\title{
Acceleration of the computation of the method of moments EFIE impedance matrix from an updated Fraunhofer criterion
}

\author{
Christophe Bourlier
}

\begin{abstract}
This paper deals with the acceleration of the computation of the impedance matrix obtained from the electric field integral equation (EFIE) discretized by the Galerkin method of moment (MoM) with Rao-Wilton-Glisson basis functions. The elements of the impedance matrix need to calculate a double integral (quadruple integral) over two planar triangles, which is typically done from two numerical Gauss-Legendre integrations. For far-field interactions, this integration can be done analytically by introducing a criterion for which the resulting closed-form expression is valid. This approximation is tested on a sphere and a concave cavity-shaped object, for which the results show that the time saving factor is about 20, with a mean difference of 0.1-0.5 dB on the radar cross section (RCS) compared to that obtained from two numerical integrations.

Index Terms-Electric Field Integral Equation, Method of Moments, Radar cross section, fast algorithm.
\end{abstract}

\section{INTRODUCTION}

The method of moments (MoM) [1] has been commonly used to solve electromagnetic scattering problems. It transforms integral equations into a matrix equation. For small problems, the resulting linear system can be solved from the LU (lower-upper) decomposition. For larger problems, iterative solvers like the conjugate gradient and their improved versions [2], [3] can be employed, in which accelerations are accounted for [4], [5]. Another family of fast iterative solvers has also been developed. The problem geometry is subdivided into sub-domains (blocks) and the problem solution is then reduced in order to successively solve a set of impedance submatrix equations [6], [7], in which accelerations are included [8], [9].

Whatever the solver, some elements of the impedance matrix must be calculated. This step requires the evaluation of a double integration (quadruple one-dimensional integral) over the source and observation facets (of triangular shapes in our case, since the Rao-Wilton-Glisson basis functions are used to discretize the electric field integral equation (EFIE) from the Garlerkin MoM). Usually, two Gauss-Legendre integrations with $n_{\text {Gauss }}$ points are applied, both on the source and observation triangles. For large $n_{\text {Gauss }}$, this step can be time consuming. The purpose of this paper is to accelerate this stage when the observation facet is in the far-field with regard to the source facet. Then, a criterion is derived by updating the conventional Fraunhofer criterion to our problem. When

C. Bourlier is with IETR (Institut d'Electronique et de Télécommunications de Rennes) laboratory, LUNAM Université, Université de Nantes, La Chantrerie, Nantes, France. this criterion is valid, it is shown that the two integrations can be done analytically, which reduces the computing time. In addition, it is important to underline that the closedform expression is only valid for planar facets and that the complexity of assembling the matrix is not changed.

The paper is organized as follows. Section 2 presents the MoM, whereas section 3 derives the criterion and gives the closed-form expression of an element of the impedance matrix. Section 4 presents numerical results, and the last section gives concluding remarks.

\section{METHOD OF MOMENTS}

In this paper, to compute the field scattered by a perfectlyconducting object, the EFIE is solved from the MoM. In addition, the Galerkin method is applied with the Rao-WiltonGlisson basis functions. This leads to solve the linear system $\bar{Z} \boldsymbol{X}=\boldsymbol{b}$, where $\bar{Z}$ is the impedance matrix, $\boldsymbol{b}$ a vector related to the incident wave and $\boldsymbol{X}$, the unknown vector. The time convention $e^{-j \omega t}$ is used throughout this paper.

The element $Z_{m, n}$ of the impedance matrix $\bar{Z}$, corresponding to the interaction between two edges $m$ (observation) and $n$ (source) of a facet couple $(p, q)$ is expressed as [2]

$$
Z_{m, n}=\frac{c_{m, n}}{A_{p} A_{q}} \iint_{\mathrm{T}_{p}} \iint_{\mathrm{T}_{q}}\left[\frac{1}{4} \boldsymbol{\rho}_{m}^{p} \cdot \boldsymbol{\rho}_{n}^{q}-\frac{1}{k^{2}}\right] \frac{e^{-j k D_{p, q}}}{D_{p, q}} d R_{p} d R_{q},
$$

where $c_{m, n}=L_{m} L_{n} s_{m, n} /(4 \pi)$, in which $\left\{L_{m, n}\right\}$ are the edge lengths and $s_{m, n}= \pm 1,\left\{A_{p, q}\right\}$ are the triangle areas, $\boldsymbol{\rho}_{m, n}^{p, q}=\boldsymbol{V}_{m, n}^{p, q}-\boldsymbol{R}_{p, q}$, in which $\boldsymbol{V}_{m, n}^{p, q}$ is the position vector of the vertex unshared by the edge $(m, n)$ and belonging to the facet $(p, q)$. In addition, $D_{p, q}=\left\|\boldsymbol{R}_{p}-\boldsymbol{R}_{q}\right\|$ and $k$ is the wavenumber which equals $2 \pi / \lambda$, where $\lambda$ is the wavelength.

Assuming a plane incident wave, a component $b_{n}$ of the vector $\boldsymbol{b}$ associated to the source edge $n$ and facet $q$ is [2]

$$
b_{n}=-\frac{j}{\omega \mu} \frac{L_{n} s_{n}}{2 A_{q}} \iint_{\mathrm{T}_{q}} \boldsymbol{\rho}_{n}^{q} \cdot \hat{\boldsymbol{p}}_{\text {inc }} e^{-\boldsymbol{k}_{\text {inc }} \cdot \boldsymbol{R}_{q}} d R_{q},
$$

where $\omega$ is the wave pulsation and $\mu$ the permeability of the surrounding medium. In addition, $\hat{\boldsymbol{p}}_{\text {inc }}$ (either vertical, $\hat{\boldsymbol{v}}_{\text {inc }}$ $(\theta)$, or horizontal, $\left.\hat{\boldsymbol{h}}_{\text {inc }}(\phi)\right)$ and $\boldsymbol{k}_{\text {inc }}$ are the polarization and incident wave vectors, respectively, both defined in spherical coordinates from the angles $\left(\theta_{\text {inc }}, \phi_{\text {inc }}\right)$. Solving the linear system $\boldsymbol{X}=\overline{\boldsymbol{Z}}^{-1} \boldsymbol{b}$, the components $\left\{a_{n}\right\}$ of the vector $\boldsymbol{X}$ 


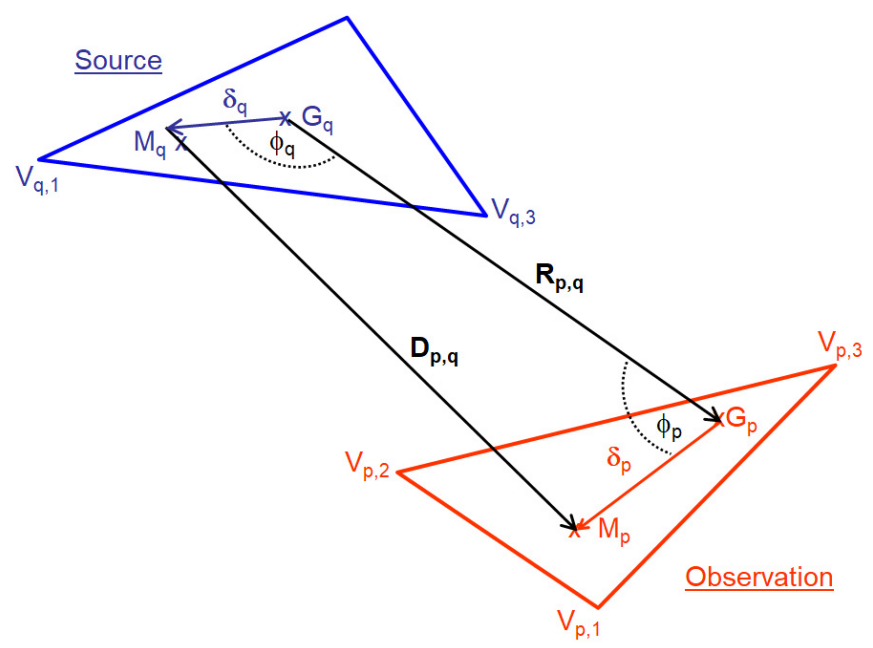

Fig. 1. Interaction of a facet source $q$ with an observation facet $p$. The point $G_{p, q}$ is the gravity centre of the triangle $(p, q)$ and the point $M_{p, q}$ is the integration point which spans the triangle $(p, q)$.

are found. The far-field scattered field is then expressed as

$$
\begin{aligned}
\boldsymbol{E}_{\mathrm{sca}}^{\infty}\left(\boldsymbol{R}_{0}\right) & =-\frac{j \omega \mu e^{-j k R_{0}}}{8 \pi R_{0}} \sum_{p=1}^{P_{\text {Facet }}} \sum_{m=1}^{M_{\mathrm{Edge}}} \frac{L_{m} a_{m} s_{m}}{A_{p}} \\
& \times \iint_{\mathrm{T}_{p}} \boldsymbol{\rho}_{m}^{p} e^{j \boldsymbol{k}_{\mathrm{sca}} \cdot \boldsymbol{R}_{p}} d R_{p},
\end{aligned}
$$

where $P_{\text {Facet }}$ is the number of facets and $M_{\text {Edge }}$ the number of edges associated to the facet $p$. In addition, $R_{0}$ is the distance from the receiver to the phase origin of the object. The scattering coefficient is then expressed as

$$
\mathrm{SC}_{p_{\text {inc }} p_{\text {sca }}}=\lim _{R_{0} \rightarrow \infty} 2 \sqrt{\pi} R_{0} \frac{\boldsymbol{E}_{\mathrm{sca}}^{\infty} \cdot \hat{\boldsymbol{p}}_{\mathrm{sca}}}{\boldsymbol{E}_{\mathrm{inc}} \cdot \hat{\boldsymbol{p}}_{\mathrm{inc}}}
$$

where $p_{\text {inc }}=\{\theta, \phi\}$ and $p_{\text {sca }}=\{\theta, \phi\}$. The subscripts "inc" and "sca" stand for incident and scattered (waves), respectively. The receiver polarization basis $\left(\hat{\boldsymbol{k}}_{\text {sca }}, \hat{\boldsymbol{v}}_{\text {sca }}, \hat{\boldsymbol{h}}_{\text {sca }}\right)$ can be defined in a similar way as that of the incident field $\left(\hat{\boldsymbol{k}}_{\text {inc }}, \hat{\boldsymbol{v}}_{\text {inc }}, \hat{\boldsymbol{h}}_{\text {inc }}\right)$, in which $\theta_{\text {sca }}$ and $\phi_{\text {sca }}$ are the receiver (scattering) angles. The radar cross section $\mathrm{RCS}_{p_{\text {inc }} p_{\text {sca }}}$ is obtained by taking the squared modulus of $\mathrm{SC}_{p_{\text {inc }} p_{\mathrm{sca}}}$.

Equation (1) shows that $Z_{m, n}$ requires the calculation of two numerical integrations over the surfaces of the triangles $\mathrm{T}_{p}$ and $\mathrm{T}_{q}$. This is done from two-fold Gauss-Legendre integrations. In this paper, we propose to derive them from a closed-form expression. The singularity, which occurs for $D_{p, q}=0$, is computed from the work published by [10].

\section{Approximation of $Z_{m, n}$}

As shown in Fig. 1, the distance $D_{p, q}=\left\|\boldsymbol{D}_{p, q}\right\|=$ $\left\|\overrightarrow{M_{q} M_{p}}\right\|$, where $\overrightarrow{M_{q} M_{p}}=\overrightarrow{M_{q} G_{q}}+\overrightarrow{G_{q} G_{p}}+\overrightarrow{G_{p} M_{p}}=\boldsymbol{R}_{p, q}+$ $\boldsymbol{\delta}$, where $\boldsymbol{R}_{p, q}=\overrightarrow{G_{q} G_{p}}$ and $\boldsymbol{\delta}=\overrightarrow{M_{q} G_{q}}+\overrightarrow{G_{p} M_{p}}=\boldsymbol{\delta}_{p}-\boldsymbol{\delta}_{q}$. If $\left\|\overrightarrow{G_{q} G_{p}}\right\|=R_{p, q} \gg\|\delta\|=\delta$, the distance $D$ can be expanded over $R_{p, q}$ up to the second order as

$$
\begin{aligned}
D_{p, q} & =\sqrt{R_{p, q}^{2}+\delta^{2}+2 R_{p, q} \delta \cos \phi} \\
& \approx R_{p, q}+\delta \cos (\phi)+\frac{\sin ^{2}(\phi) \delta^{2}}{2 R_{p, q}}
\end{aligned}
$$

where $\phi=\left(\widehat{\boldsymbol{R}_{p, q}}, \boldsymbol{\delta}\right)$. The term in $\delta$ is related to the local behavior of a plane wave, whereas $\delta^{2}$ is related to the local behavior of a spherical wave. The Fraunhofer criterion is obtained from equation (5) by neglecting the term in $\delta^{2}$. In Green's function, this approximation is satisfied if $\delta^{2} \sin ^{2} \phi /\left(2 R_{p, q}\right)$ does not exceed $\lambda / n_{0}$ (typically $n_{0}$ is an integer ranging from 10 to 20). This leads for $k D_{p, q}$, to

$$
\frac{\delta^{2}}{2} \frac{\sin ^{2} \phi}{R_{p, q}} \leq \frac{\lambda}{n_{0}}
$$

The maximum value of $\delta$, named $\Delta$, equals

$$
\begin{aligned}
\Delta & =\max \left\|\boldsymbol{\delta}_{p}-\boldsymbol{\delta}_{q}\right\|=\max \left\|\boldsymbol{\delta}_{p}\right\|+\max \left\|\boldsymbol{\delta}_{q}\right\| \\
& \approx \sum_{i=\{p, q\}} \max \left[\left(x_{M_{i}}-x_{G_{i}}\right)^{2}+\left(y_{M_{i}}-y_{G_{i}}\right)^{2}\right. \\
& \left.+\left(z_{M_{i}}-z_{G_{i}}\right)^{2}\right]^{1 / 2} .
\end{aligned}
$$

If the criterion (7) is satisfied, then

$$
D \approx R_{p, q}+\delta \cos (\phi)=R_{p, q}+\frac{\boldsymbol{R}_{p, q}}{R_{p, q}} \cdot\left(\boldsymbol{\delta}_{p}-\boldsymbol{\delta}_{q}\right) .
$$

In equation (1), the double integral can be simplified as

$$
\begin{aligned}
I_{m, n} & =\frac{1}{A_{p} A_{q}} \iint_{\mathrm{T}_{p}} \iint_{\mathrm{T}_{q}}\left[\frac{1}{4} \boldsymbol{\rho}_{m}^{p} \cdot \boldsymbol{\rho}_{n}^{q}-\frac{1}{k^{2}}\right] \frac{e^{-j k D p, q}}{D_{p, q}} d R_{p} d R_{q} \\
& \approx \frac{e^{-j k R_{p, q}}}{A_{p} A_{q} R_{p, q}} \iint_{\mathrm{T}_{p}} \iint_{\mathrm{T}_{q}}\left[\frac{1}{4} \boldsymbol{\rho}_{m}^{p} \cdot \boldsymbol{\rho}_{n}^{q}-\frac{1}{k^{2}}\right] \\
& \times e^{-j k \hat{\boldsymbol{R}}_{p, q} \cdot \boldsymbol{\delta}_{p}} e^{+j k \hat{\boldsymbol{R}}_{p, q} \cdot \boldsymbol{\delta}_{q}} d R_{p} d R_{q}
\end{aligned}
$$

where $\hat{\boldsymbol{R}}_{p, q}=\boldsymbol{R}_{p, q} / R_{p, q}$. Using the variable transformation $\boldsymbol{\delta}_{p}=\overrightarrow{G_{p} M_{p}}=\overrightarrow{G_{p} O}+\overrightarrow{O M_{p}}=\overrightarrow{O M_{p}}-\overrightarrow{O G_{p}}=\overrightarrow{O M_{p}}-$ $\boldsymbol{R}_{G_{p}} \Rightarrow \overrightarrow{O M_{p}}=\boldsymbol{R}_{p}=\boldsymbol{\delta}_{p}+\boldsymbol{R}_{G_{p}}$, where the point $O$ stands for the phase origin, the above integral can be simplified as

$$
I_{m, n}=\frac{e^{-j k R_{p, q}}}{R_{p, q}}\left[\frac{1}{4} \boldsymbol{J}_{q, m} \cdot \boldsymbol{J}_{p, n}^{*}-\frac{1}{k^{2}} K_{q} K_{p}^{*}\right],
$$

where

$$
K_{i}=\frac{1}{A_{i}} \iint_{\mathrm{T}_{i}^{\prime}} e^{j k \hat{\boldsymbol{R}}_{p, q} \cdot \boldsymbol{\delta}_{i}} d \delta_{i},
$$

and

$$
\begin{aligned}
\boldsymbol{J}_{i, m} & =\frac{1}{A_{i}} \iint_{\mathrm{T}_{i}^{\prime}}\left(\boldsymbol{V}_{m}^{i}-\boldsymbol{R}_{G_{i}}-\boldsymbol{\delta}_{i}\right) e^{j k \hat{\boldsymbol{R}}_{p, q} \cdot \boldsymbol{\delta}_{i}} d \delta_{i} \\
& =\left(\boldsymbol{V}_{m}^{i}-\boldsymbol{R}_{G_{i}}\right) K_{i}-\boldsymbol{L}_{i},
\end{aligned}
$$

where

$$
\boldsymbol{L}_{i}=\frac{1}{A_{i}} \iint_{\mathrm{T}_{i}^{\prime}} \boldsymbol{\delta}_{i} e^{j k \hat{\boldsymbol{R}}_{p, q} \cdot \boldsymbol{\delta}_{i}} d \delta_{i} .
$$

It is important to underline that the integration domain $\mathrm{T}_{i}^{\prime}$ of the facet $i=(p, q)$ is now defined from the gravity centre $\boldsymbol{R}_{G_{i}}$. In addition, the integrals $K_{i}$ (scalar) and $\boldsymbol{L}_{i}$ (vector) do 
not depend on the edge and only depend on the distance $R_{p, q}$ and on the triangle $i$ defined from its three vertices. Owing to the presence of the term $\boldsymbol{V}_{m}^{i}$, the integral $\boldsymbol{J}_{i, m}$ depends on the edge $m$. As shown in appendix, the integrals $K_{i}$ and $\boldsymbol{L}_{i}$ can be derived analytically. In conclusion, an element of the impedance matrix can be computed analytically from equation (10) if

$$
\frac{\Delta^{2}}{2} \frac{\sin ^{2} \phi}{R_{p, q}} \approx \frac{\Delta^{2}}{2 R_{p, q}} \leq \frac{\lambda}{n_{0}} .
$$

In practice, the angle $\phi$ can not be determined numerically since it depends on the integration variables. Then, $\phi=\pi / 2$ is chosen as an upper limit of the criterion. For two given triangles $p$ and $q$, the coordinates of their gravity centers $\left\{\boldsymbol{R}_{G_{p}}, \boldsymbol{R}_{G_{q}}\right\}$ are known and then the distance $R_{p, q}=$ $\left\|\boldsymbol{R}_{G_{p}}-\boldsymbol{R}_{G_{q}}\right\|$ is computed. Moreover, $\Delta$ is computed from equation (7). Typically, $\Delta=a_{p}+a_{q}$, where $a_{i}$ is the circle radius circumscribed to the triangle $i(i=\{p, q\})$.

It is important to underline that integrals (2) and (3) can also be computed analytically, since

$$
\frac{1}{A_{p}} \iint_{\mathrm{T}_{i}} \boldsymbol{\rho}_{m}^{i} e^{\boldsymbol{s} \cdot \boldsymbol{R}_{i}} d R_{i}=\boldsymbol{V}_{m}^{i} K_{i}-\boldsymbol{L}_{i} .
$$

For equation (2), $s=-\boldsymbol{k}_{\text {inc }}$ and for equation (3), $\boldsymbol{s}=\boldsymbol{k}_{\text {sca }}$. In addition, the variable transformation over $\boldsymbol{\delta}_{i}$ is not applied (the three vertices of the triangle $i$ are defined from the phase origin $O$ ).

\section{NUMERICAL RESUlTS}

The wavelength in free space $\lambda$ is $1 \mathrm{~m}$ and the polarization is $\theta \theta$.

First, to test the accuracy of the proposed method, a sphere is considered, for which the field scattered by a plane wave is known exactly from the Mie series [11].

Fig. 2 plots the bistatic RCS in $\mathrm{dBm}^{2}$ versus the scattering angle $\theta_{\text {sca. }}$. The incidence angles are $\theta_{\text {inc }}=0, \phi_{\text {inc }}=0$ and $\phi_{\text {sca }}=0$ is the scattering azimuthal angle. To better highlight the differences, Fig. 3 plots the corresponding ratio $\mathrm{RCS} /\left.\mathrm{RCS}\right|_{\text {Mie }}$ in $\mathrm{dB}$ scale (becomes a difference) versus the scattering angle $\theta_{\text {sca }}$, where $\left.\mathrm{RCS}\right|_{\text {Mie }}$ is the reference Mie solution. The sphere has a radius $a=0.6 \lambda_{0}$ and the number of edges is $N_{\text {Edge }}=6279$, corresponding to an edge mean length of $0.05 \lambda_{0}$. As shown in Figs. 2 and 3, this value ensures a very good agreement between the results obtained from two Gauss-Legendre integrations, for which the number of points is $n_{\text {Gauss }}=6$.

In Fig. 3, in the legend, the first number gives the mean value of $10\left|\log _{10}\left(\mathrm{RCS} /\left.\mathrm{RCS}\right|_{\text {Mie }}\right)\right|$ over $\theta_{\text {sca }} \in[0 ; \pi]$. The acronym "UFC" means updated Fraunhofer criterion. The second number $p$ gives the percentage of facet pairs which are in far-field and for which the approximation is applied. The integer $n_{0}$ is the number of points per wavelength, corresponding to a phase error of $\lambda_{0} / n_{0}$ to estimate Green's function. As expected, as $n_{0}$ increases, the results better match with those of Mie and are very similar to those computed from two numerical integrations.

In Fig. 2, in the legend, the first number $t$ gives the computing time to calculate the impedance matrix. In comparison



Fig. 2. Bistatic RCS in $\mathrm{dBm}^{2}$ versus the scattering angle $\theta_{\text {sca. }} \theta_{\text {inc }}=0$, $\phi_{\text {inc }}=0$ and $\phi_{\text {sca }}=0$. The illuminated object is a sphere of radius $a=$ $0.6 \lambda_{0}$.

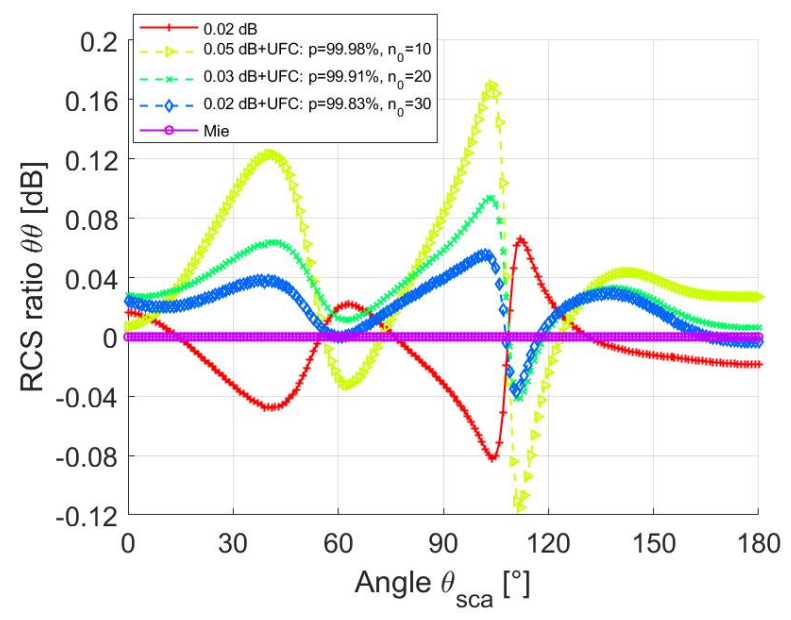

Fig. 3. Ratio RCS $/\left.\mathrm{RCS}\right|_{\text {Mie }}$ in $\mathrm{dB}$ scale versus the scattering angle $\theta_{\text {sca }}$. The parameters are the same as in Fig. 2.
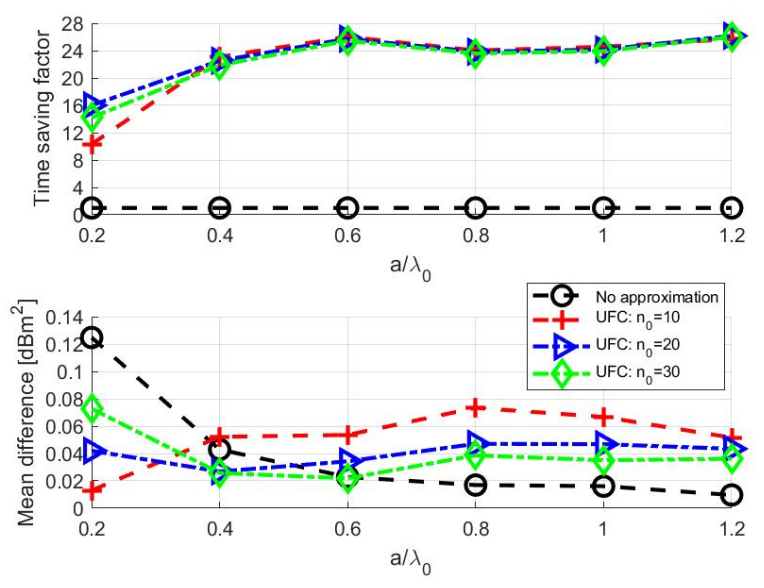

Fig. 4. Top: Time saving factor versus $a / \lambda_{0}$. Bottom: Mean difference on the RCS ratio versus $a / \lambda_{0}$. 


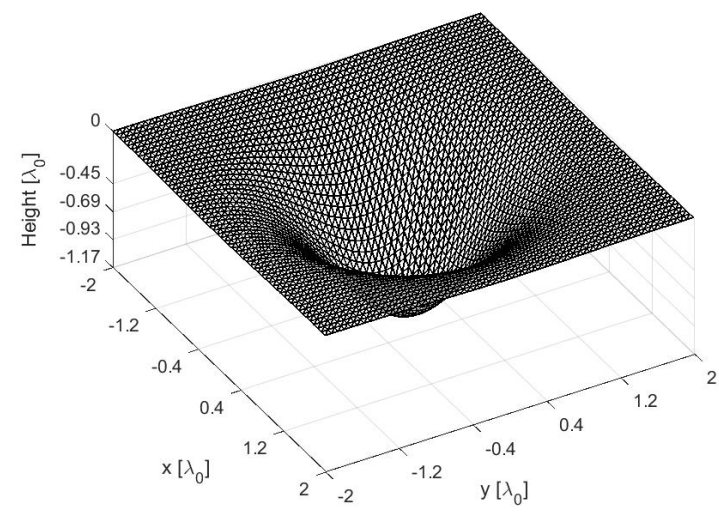

Fig. 5. Example of a concave cavity-shaped object with $L_{x}=L_{y}=4 \lambda_{0}$. The number of edges is $N_{\text {Edge }}=9633$ and the edge mean length is $0.08 \lambda_{0}$.

to two numerical integrations, the time saving factor is of the order of 25 . As $n_{0}$ decreases, the number of the facet pairs $p$ in far-field increases and then, all the elements of the impedance matrix are computed from the approximation. The time saving factor is approximatively proportional to $\eta_{t}=n_{\text {Gauss }}^{2} / C_{\mathrm{App}}$, where $n_{\text {Gauss }}^{2}$ is the complexity of two numerical integrations and $C_{\mathrm{App}}$, that of the approximation. Theoretically, this value is equal to one, but it differs from one in practice because the calculation of the analytical expression needs some multiplications. This explains why in Fig. $2, \eta_{t}$ is smaller than $n_{\text {Gauss }}^{2}=6^{2}=36$. Fig. 2 also shows that $\eta_{t}$ is little sensitive to $n_{0}$.

Fig. 4 plots the time saving factor $\eta_{t}$ versus $a / \lambda_{0}$ ( $N_{\text {Edge }}$ ranging from 669 to 24,321) and at the bottom, the mean ratio $10\left|\log _{10}\left(\mathrm{RCS} /\left.\mathrm{RCS}\right|_{\text {Mie }}\right)\right|$ over $\theta \in[0 ; \pi]$ is plotted versus $a / \lambda_{0}$. As the radius $a$ increases, the gain in time saving is nearly constant, equal to approximately 25 , and the mean difference does not exceed $0.08 \mathrm{~dB}$. The percentage of facet pairs in far-field over $a / \lambda_{0} \in[0.2,1.2]$ ranges from $\{0.9836,0.9919,0.9978\}$ to $\{0.9995,0.9998,0.9999\}$ for $n_{0}=\{30,20,10\}$, respectively.

To produce strong interactions between the facets, a concave cavity-shaped object is considered to produce multiple reflections. The geometry is shown in Fig. 5. It is defined as $z(x, y)=-A \exp \left(-x^{2} / a_{1}^{2}-y^{2} / b_{1}^{2}\right)$ where $a_{1}=L_{x} / 4$, $b_{1}=L_{y} / 4, A=a_{1} e^{1 / 2} / \sqrt{2}$ and $L_{x}$ and $L_{y}$ are the lengths of the object (defined for $z=0$ corresponding to the top of figure 5) with respect to the $x$ and $y$ directions, respectively. In addition, the number $A$ is chosen such that the absolute values of the maximum slopes with respect to the $x$ and $y$ directions are equal to one in order to produce a dihedral effect (at least two reflections).

Fig. 6 plots the RCS in $\mathrm{dBm}^{2}$ versus the scattering angle $\theta_{\text {sca. }} \theta_{\text {inc }}=0, \phi_{\text {inc }}=0$ and $\phi_{\text {sca }}=0$. Fig. 7 plots the ratio $\mathrm{RCS} /\left.\mathrm{RCS}\right|_{0}$ in $\mathrm{dB}$ scale versus the scattering angle $\theta_{\text {sca }}$. The parameters are the same as in Fig. 6 and the number RCS $\left.\right|_{0}$ is the RCS calculated without approximation.

Fig. 6 shows a very good agreement between the RCS computed without approximation and those obtained from the

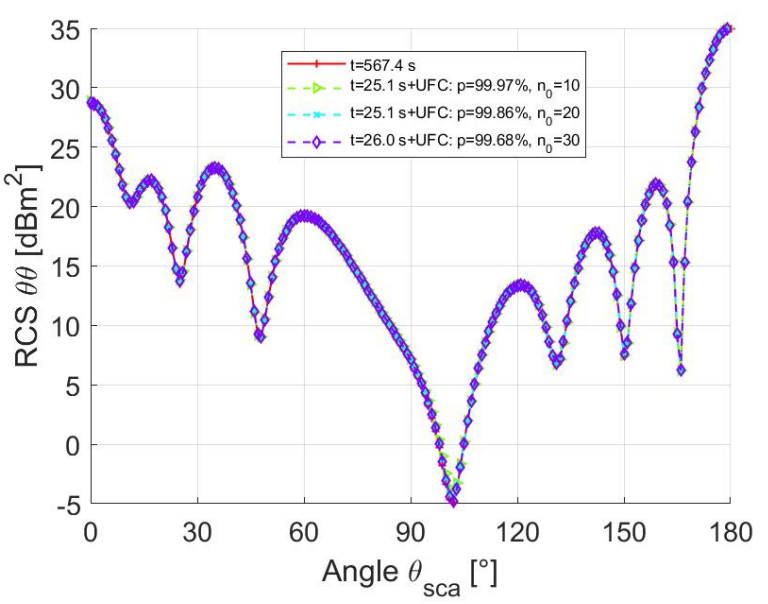

Fig. 6. RCS in $\mathrm{dBm}^{2}$ versus the scattering angle $\theta_{\text {sca. }} \cdot \theta_{\text {inc }}=0, \phi_{\text {inc }}=0$ and $\phi_{\text {sca }}=0$. A concave cavity-shaped object is considered (Fig. 5).

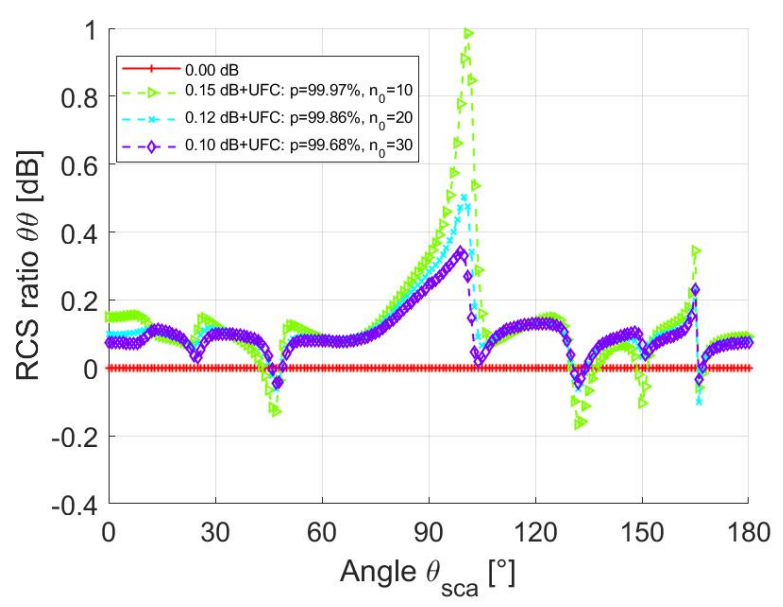

Fig. 7. Ratio RCS $/\left.\mathrm{RCS}\right|_{0}$ in $\mathrm{dB}$ scale versus the scattering angle $\theta_{\text {sca. }}$. The parameters are the same as in Fig. 6.
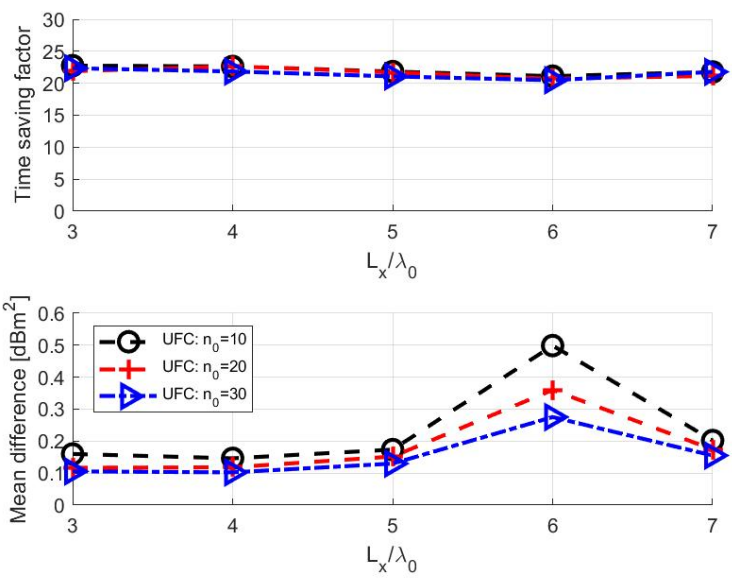

Fig. 8. Top: Time saving factor versus $L_{x} / \lambda_{0}\left(L_{y}=L_{x}\right)$. Bottom: Mean difference on the RCS ratio versus $a / \lambda_{0}$. 
approximation. Like for the sphere, the time saving factor is of the order of 21. In Fig. 7, the difference decreases as $n_{0}$ increases, and it increases as the RCS level in Fig. 6 takes low values. For these particular values, a better precision is required.

Fig. 8 plots the time saving factor versus $L_{x} / \lambda_{0}\left(L_{y}=\right.$ $L_{x}$ and $N_{\text {Edge }}$ ranging from 5208 to 29800). At the bottom, the RCS ratio between the proposed method and that without approximation is plotted in $\mathrm{dB}$ scale (becomes a difference) versus $L_{x} / \lambda_{0}$. As we can see, the gain in time saving is little sensitive to the length $L_{x}$ and the mean difference does not exceed $0.5 \mathrm{~dB}$. As $n_{0}$ increases, this value slightly decreases.

\section{CONCLUSION}

In this paper, from the derivation of a far-field criterion, the elements of the EFIE impedance matrix are computed from a closed-form expression. This approximation avoids to calculate these elements from two numerical integrations, which can be time consuming. The numerical results showed that the proposed method offers a time saving factor of the order of 25 for $n_{\text {Gauss }}=6$, whereas for $n_{\text {Gauss }}=3$ it equals 6 (not shown in the paper). This number is directly related to $n_{\text {Gauss }}^{2}$, which corresponds to the complexity of the two numerical integrations. The choice of $n_{\text {Gauss }}$ depends on the expected accuracy (related to the surface curvature, calculation in near or far field, ...). In addition, for the $\phi \phi$ polarization, results (not depicted here) show that the approximation gives better results in comparison to the $\theta \theta$ polarization.

\section{APPENDIX A}

\section{DERIVATION OF THE INTEGRAL FOR A TRIANGULAR SHAPE}

This appendix presents the derivation of the double integral (9).

In simplex coordinates, any two-dimensional integral on a triangular domain $\mathrm{T}$ can be converted as (Eq. (9.29) of [2])

$$
\iint_{\mathrm{T}} f(\boldsymbol{R}) d R=2 A \int_{0}^{1} \int_{0}^{1-\alpha} f(\alpha, \beta) d \alpha d \beta
$$

where $\boldsymbol{R}=(1-\alpha-\beta) \boldsymbol{V}_{1}+\alpha \boldsymbol{V}_{2}+\beta \boldsymbol{V}_{3}$ and $\left(\boldsymbol{V}_{1}, \boldsymbol{V}_{2}, \boldsymbol{V}_{3}\right)$ are the coordinates of the three vertices of the triangle $\mathrm{T}$ of area $A$. Then

$$
\begin{aligned}
K(\boldsymbol{s}) & =\frac{1}{A} \iint_{\mathrm{T}} e^{j \boldsymbol{s} \cdot \boldsymbol{R}} d R=2 e^{j \boldsymbol{s} \cdot \boldsymbol{V}_{1}} \\
& \times \int_{0}^{1} \int_{0}^{1-\alpha} e^{j \boldsymbol{s} \cdot\left(\boldsymbol{V}_{2}-\boldsymbol{V}_{1}\right) \alpha} e^{j \boldsymbol{s} \cdot\left(\boldsymbol{V}_{3}-\boldsymbol{V}_{1}\right) \beta} d \alpha d \beta,
\end{aligned}
$$

where $s$ is a constant vector (independent of $\boldsymbol{R}$ ). After some tedious but straightforward algebra, we obtain (equation (9.44) of [2])

$$
K(\boldsymbol{s})=\frac{2 A e^{j s_{1}}}{s_{3}-s_{2}}\left(\frac{1-e^{j s_{3}}}{s_{3}}-\frac{1-e^{j s_{2}}}{s_{2}}\right) .
$$

where $s_{1}=\boldsymbol{s} \cdot \boldsymbol{V}_{1}, s_{2}=\boldsymbol{s} \cdot\left(\boldsymbol{V}_{2}-\boldsymbol{V}_{1}\right)$ and $s_{3}=\boldsymbol{s} \cdot\left(\boldsymbol{V}_{3}-\boldsymbol{V}_{1}\right)$. In addition, since $e^{j s} \approx 1+j s$ for $|s| \ll 1$, we can show that

$$
K(s)=2 e^{j s_{1}} \begin{cases}\left(1+j s_{2}-e^{j s_{2}}\right) / s_{2}^{2} & \text { if } s_{3}=0 \\ \left(1+j s_{3}-e^{j s_{3}}\right) / s_{3}^{2} & \text { if } s_{2}=0 \\ e^{j s_{3}}\left[\left(1-j s_{3}\right)-1\right] / s_{3}^{2} & \text { if } s_{2}=s_{3} \\ 1 / 2 & \text { if } s_{2}=s_{3}=0\end{cases}
$$

The above equation is useful to avoid numerical problems. Applying the same way for $\boldsymbol{L}(\boldsymbol{s})$, we show that

$$
\begin{aligned}
\boldsymbol{L}(\boldsymbol{s}) & =\iint_{\mathrm{T}} \boldsymbol{R} e^{j \boldsymbol{s} \cdot \boldsymbol{R}} d R=2 A e^{j s_{1}} \int_{0}^{1} \int_{0}^{1-\alpha} d \alpha d \beta \\
& {\left[(1-\alpha-\beta) \boldsymbol{V}_{1}+\alpha \boldsymbol{V}_{2}+\beta \boldsymbol{V}_{3}\right] e^{j s_{2} \alpha} e^{j s_{3} \beta} } \\
= & \boldsymbol{V}_{1} F_{0}(\boldsymbol{s})-j\left(\boldsymbol{V}_{2}-\boldsymbol{V}_{1}\right) \frac{\partial K}{\partial s_{2}}-j\left(\boldsymbol{V}_{3}-\boldsymbol{V}_{1}\right) \frac{\partial K}{\partial s_{3}} .
\end{aligned}
$$

From Eq. (A3), the partial derivatives are expressed as

$$
\left\{\begin{array}{l}
\frac{\partial K}{\partial s_{2}}=\frac{2 A e^{j s_{1}}}{s_{3}-s_{2}}\left[\frac{K(s)}{2 A e^{j s_{1}}}+\frac{1+e^{j s_{2}}\left(j s_{2}-1\right)}{s_{2}^{2}}\right] \\
\frac{\partial K}{\partial s_{3}}=\left.\frac{\partial K}{\partial s_{2}}\right|_{\left(s_{2}, s_{3}\right) \rightarrow\left(s_{3}, s_{2}\right)}
\end{array}\right.
$$

In addition

$$
\frac{\partial K}{\partial s_{2}}=2 e^{j s_{1}} \begin{cases}{\left[-2-j s_{2}+e^{j s_{2}}\left(2-j s_{2}\right)\right] / s_{2}^{3}} & \text { if } s_{3}=0 \\ \left.2+2 j s_{3}-s_{3}^{2}-2 e^{j s_{3}}\right] / s_{3}^{2} & \text { if } s_{2}=0 \\ \left.2+e^{j s_{3}}\left(s_{3}^{2}+2 j s_{3}-2\right)\right] /\left(2 s_{3}^{3}\right) & \text { if } s_{2}=s_{3} \\ j / 6 & \text { if } s_{2}=s_{3}=0\end{cases}
$$

In conclusion, $K_{i}$ expressed from equation (11) is obtained from equation (A3) by setting $s=k \hat{\boldsymbol{R}}_{p, q}$, for which the triangle $i$ is defined from its three vertices. In addition, $\boldsymbol{L}_{i}$ (equation (13)) is obtained from equation (A5) by setting $s=k \hat{\boldsymbol{R}}_{p, q}$ and $\boldsymbol{\delta}_{i}=\boldsymbol{R}$, for which the three vertices of the facet $i$ are defined from their gravity centre $\boldsymbol{R}_{G_{i}}$.

\section{REFERENCES}

[1] R. F. Harrington, Field Computation by Moment Method. New York: Macmillan, 1968.

[2] W. C. Gibson, The Method of Moments in Electromagnetics, Chapman and Hall/CRC, Eds., London, 2008.

[3] A. Quarteroni, R. Sacco, and F. Sameri, Méthodes Numériques: Algortihmes, analyse et Applications. New-York: Springer-Verlag, 2007.

[4] R. Coifman, V. Rokhlin, and S. Wandzura, "The fast multipole method for the wave equation: a pedestrian prescription," IEEE Antennas and Propagation Magazine, vol. 35, no. 3, pp. 7-12, 1993.

[5] C.-C. Lu and W. C. Chew, "Fast far field approximation for calculating the RCS of large objects," Microwave Optical Technology Letter, vol. 8, no. 5 , pp. $238-241,1995$.

[6] V. S. Prakash and R. Mitra, "Characteristic Basis Function Method: A new technique for efficient solution of Method of Moments matrix equations," Microwave Optical Technology Letter, vol. 26, no. 2, pp. 95-100, 2003.

[7] C. Bourlier, S. Bellez, and G. Kubické, "Sub-Domain Decomposition Iterative method combined with ACA: An efficient technique for the scattering from a large highly conducting rough sea surface," IEEE Transactions on Antennas and Propagation, vol. 63, no. 2, pp. 659666, 2015.

[8] K. Zhao, M. N. Vouvakis, and J.-F. Lee, "The adaptive cross approximation algorithm for accelerated method of moments computations of EMC problems," IEEE Transactions on Electromagnetic Compatibility, vol. 47, no. 4, pp. 763-773, 2005.

[9] C. Li and R. Mittra, "Characteristic basis function method for fast analysis of 3-D scattering from objects buried under rough surfaces," IEEE Transactions on Geoscience and Remote Sensing, vol. 57, no. 8, pp. 5252-5265, 2019.

[10] W. T. Sheng, Z. Y. Shu, K. Yang, and M. S. Tong, "Efficient evaluation of weakly singular integrals arising from electromagnetic surface integral equations," IEEE Transactions on Antennas and Propagation, vol. 61, no. 6, pp. 3377-3381, 2013.

[11] L. Tsang, J. A. Kong, and K.-H. Ding, Scattering of Electromagnetic Waves, Theories and Applications, W. S. in Remote Sensing, Ed. John Wiley \& Sons, Inc., 2000. 